\title{
Calculation of magnetic oscillations via the magnetic-field-containing relativistic tight-binding approximation method: Revisiting the de Haas-van Alphen effect
}

\author{
Dipendra Bahadur Hamal, ${ }^{1}$ Masahiko Higuchi, ${ }^{2}$ and Katsuhiko Higuchi ${ }^{1}$ \\ ${ }^{1}$ Graduate School of Advanced Sciences of Matter, Hiroshima University, Higashi-Hiroshima 739-8527, Japan \\ ${ }^{2}$ Department of Physics, Faculty of Science, Shinshu University, Matsumoto 390-8621, Japan \\ (Received 28 February 2015; revised manuscript received 18 April 2015; published 1 June 2015)
}

\begin{abstract}
The magnetic-field-containing relativistic tight-binding approximation (MFRTB) method [Phys. Rev. B 91, 075122 (2015)] is the first-principles calculation method for electronic structures of materials immersed in the magnetic field. In this paper, the MFRTB method is applied to the simple cubic lattice immersed in the magnetic field. The total energy and magnetization oscillate with the inverse of the magnitude of the magnetic field, which means that the de Haas-van Alphen oscillation is revisited directly through the MFRTB method. It is shown that the conventional Lifshitz-Kosevich (LK) formula is a good approximation to the results of the MFRTB method in the experimentally available magnetic field. Furthermore, the additional oscillation peaks of the magnetization are found especially in the high magnetic field, which cannot be explained by the LK formula.
\end{abstract}

DOI: 10.1103/PhysRevB.91.245101

PACS number(s): 71.15.-m

\section{INTRODUCTION}

The de Haas-van Alphen (dHvA) effect [1-3] is an oscillatory behavior of the magnetization as a function of the magnetic field. Measurements of the dHvA effect are widely used to probe the geometry of the Fermi surface, the cyclotron effective mass, and the scattering lifetime of the conduction electrons [4]. The magnetic oscillation was first discussed by Landau [5] independently of the experiments by de Haas and van Alphen. In the formulation, the oscillatory behavior of the magnetization is described by means of quantized energy levels (Landau levels) that are obtained by solving the Schrödinger equation for a free electron immersed in a uniform magnetic field [5]. However, the formula cannot explain the dependence of the magnetic oscillation on the direction of the magnetic field that is experimentally observed [6]. This is due to an oversimplified argument such that the characteristics of individual metals are not taken into consideration except for the electron density [6].

More realistic theories for the dHvA effect was developed by Onsager in 1952 [7] as well as by Lifshitz and Kosevich in 1956 [8]. The latter yields the so-called Lifshitz-Kosevich (LK) formula, which is recognized as the standard formula for explaining the dHvA effect $[4,6]$. The LK formula is based on the assumption that the orbital motion of the electron is quantized even when the magnetic field is applied to the crystalline solids. This quantization is derived by using both the Bohr-Sommerfeld quantization rule and the semiclassical equation of motion for the Bloch electron in the presence of the magnetic field [4,6-8]. Corresponding to the orbital quantization, the energy levels of the electron are also quantized. In the LK formulation, the dHvA effect is explained from such quantized energy levels: When the quantized energy levels cross the Fermi surface by increasing the magnetic field, every time one energy level matches with the Fermi energy, one oscillation of the magnetization is produced [4,6-8]. The LK formula is commonly used in estimating the extremal cross section of the Fermi surface, cyclotron effective mass, and scattering lifetime of electrons from the experimental data of the dHvA effect [9].

As mentioned above, in the LK formula, both the BohrSommerfeld quantization rule and the semiclassical equation of motion for the Bloch electron in the presence of the magnetic field are used to achieve quantized energy levels of the electrons $[4,6,8]$. On the one hand, it would be desirable or natural to get quantized energy levels of the electron by solving the Schrödinger equation or Dirac equation for the electron in both periodic potential and magnetic field $[6,10]$. However, unfortunately, it has been difficult to solve them directly $[6,10]$. Most recently, we have developed the magnetic-fieldcontaining relativistic tight-binding approximation (MFRTB) method that enables us to calculate electronic structures of materials immersed in a uniform magnetic field [11]. This method is the first-principles calculation method that is applicable to various kinds of realistic materials immersed in a uniform magnetic field [11]. Therefore, it is expected that the MFRTB method can revisit the dHvA effect from the viewpoint of the first-principles calculation. Besides the description of the dHvA effect, the MFRTB method is also expected to be used for revealing the mechanism of the elastic softening of the boron-doped silicon immersed in the magnetic field [12-15], and for solving the Kohn-Sham equations of the current-density functional theories [16-22]. In the previous paper, we have applied this method to the crystalline silicon immersed in the magnetic field and revealed the specific structures of the energy spectrum [11].

In this paper, we revisit the dHvA effect by means of the MFRTB method and check the validity of the LK formula. For this purpose, we apply the MFRTB method to a hypothetical simple cubic lattice immersed in a uniform magnetic field. The reason why a hypothetical simple cubic lattice is chosen as a test system is that the extremal cross section of the Fermi surface can be obtained exactly, which enables us to check the validity of the LK formula itself. As shown later, the dHvA effect can successfully be revisited by means of the MFRTB method. In addition, we will discuss the validity of the LK formula by comparing the magnetic oscillation obtained by the MFRTB method with that calculated on the basis of the LK formula. Furthermore, it will be shown that additional oscillation peaks, which cannot be predicted by the LK formula, appear in the magnetization.

The paper is organized as follows. A brief description of the MFRTB method [11] is presented in Sec. II A. The application 
of the MFRTB method to the simple cubic lattice immersed in a magnetic field is explained in Sec. II B. The resultant simultaneous equation that is derived with the aid of the magnetic Bloch theorem is also given in this subsection. In Sec. III, $E$ - $\boldsymbol{k}$ curves over the magnetic first Brillouin zone, density of states, and magnetic oscillations of the total energy and magnetization are presented with the comparison to the magnetic oscillation predicted by the LK formula. Finally, the concluding remarks are presented in Sec. IV.

\section{CALCUlation METHOD}

\section{A. MFRTB method}

In this subsection, we briefly review the MFRTB method that has recently been developed [11]. The Dirac equation for an electron that moves in a uniform magnetic field and periodic potential of the crystal is given by

$$
\begin{aligned}
& {\left[c \alpha \cdot\{\boldsymbol{p}+e \boldsymbol{A}(\boldsymbol{r})\}+\beta m c^{2}+\sum_{n} \sum_{i} V_{a_{i}}\left(\boldsymbol{r}-\boldsymbol{R}_{n}-\boldsymbol{d}_{i}\right)\right] \Phi_{\boldsymbol{k}}(\boldsymbol{r})} \\
& \quad=E(\boldsymbol{k}) \Phi_{\boldsymbol{k}}(\boldsymbol{r}),
\end{aligned}
$$

where $\boldsymbol{R}_{n}$ and $\boldsymbol{d}_{i}$ denote the translation vector of the lattice and vector specifying the position of atom $a_{i}$, respectively, and where $\boldsymbol{A}(\boldsymbol{r})$ and $V_{a_{i}}\left(\boldsymbol{r}-\boldsymbol{R}_{n}-\boldsymbol{d}_{i}\right)$ are the vector potential of a uniform magnetic field and scalar potential of the atom $a_{i}$ that is located at $\boldsymbol{R}_{n}+\boldsymbol{d}_{i}$, respectively. The vector $\boldsymbol{k}$ denotes the wave vector that is defined through the magnetic Bloch theorem [11], which is mentioned in Sec. II B. We suppose that the magnetic field is applied along the $z$ axis and that the Landau gauge is chosen for $\boldsymbol{A}(\boldsymbol{r})$, i.e.,

$$
\boldsymbol{A}(\boldsymbol{r})=(0, B x, 0),
$$

where $\boldsymbol{B}(\boldsymbol{r})=\nabla \times \boldsymbol{A}(\boldsymbol{r})$. The wave function $\Phi_{\boldsymbol{k}}(\boldsymbol{r})$ is expanded by using the relativistic atomic orbitals $\psi_{\xi}^{a_{i}, \boldsymbol{R}_{n}+\boldsymbol{d}_{i}}(\boldsymbol{r})$ as the basis function, i.e.,

$$
\Phi_{\boldsymbol{k}}(\boldsymbol{r})=\sum_{\xi} \sum_{n} \sum_{i} C_{\boldsymbol{k}}^{\xi}\left(\boldsymbol{R}_{n}+\boldsymbol{d}_{i}\right) \psi_{\xi}^{a_{i}, \boldsymbol{R}_{n}+\boldsymbol{d}_{i}}(\boldsymbol{r}),
$$

where $C_{\boldsymbol{k}}^{\xi}\left(\boldsymbol{R}_{n}+\boldsymbol{d}_{i}\right)$ denotes the expansion coefficient, and $\psi_{\xi}^{a_{i}, \boldsymbol{R}_{n}+\boldsymbol{d}_{i}}(\boldsymbol{r})$ obeys the following Dirac equation for the isolated atom $a_{i}$ :

$$
\begin{aligned}
& {\left[c \alpha \cdot\{\boldsymbol{p}+e \boldsymbol{A}(\boldsymbol{r})\}+\beta m c^{2}+V_{a_{i}}\left(\boldsymbol{r}-\boldsymbol{R}_{n}-\boldsymbol{d}_{i}\right)\right] \psi_{\xi}^{a_{i}, \boldsymbol{R}_{n}+\boldsymbol{d}_{i}}(\boldsymbol{r})} \\
& \quad=\varepsilon_{\xi}^{\boldsymbol{R}_{n}+\boldsymbol{d}_{i}} \psi_{\xi}^{a_{i}, \boldsymbol{R}_{n}+\boldsymbol{d}_{i}}(\boldsymbol{r}) .
\end{aligned}
$$

Matrix elements of the Hamiltonian are calculated by employing the perturbation theory, in which $c e \alpha \cdot \boldsymbol{A}(\boldsymbol{r})$ in Eq. (4) is treated as the perturbation term [11]. We also neglect the integrals involving three different centres. The resultant matrix elements in the MFRTB method are given by [11]

$$
\begin{aligned}
& H_{\boldsymbol{R}_{m} j\left(n^{\prime} l^{\prime} J^{\prime} M^{\prime}\right), \boldsymbol{R}_{n} i(n l J M)} \\
& =\left(\bar{\varepsilon}_{n l J}^{a_{i}}(\boldsymbol{B}=0)+\Delta \bar{\varepsilon}_{n l J M}^{a_{i}, \boldsymbol{d}_{i}}(\boldsymbol{B}=0)+\frac{e B}{2 m} \frac{2 J+1}{2 l+1} \hbar M\right) \\
& \quad \times \delta_{\boldsymbol{R}_{m}, \boldsymbol{R}_{n}} \delta_{j, i} \delta_{n^{\prime} l^{\prime} J^{\prime} M^{\prime}, n l J M} \\
& \quad+\left(1-\delta_{\boldsymbol{R}_{m}, \boldsymbol{R}_{n}} \delta_{j, i}\right) e^{-i \frac{e B}{2 \hbar}\left(R_{n x}+d_{i x}-R_{m x}-d_{j x}\right)\left(R_{n y}+d_{i y}+R_{m y}+d_{j y}\right)} \\
& \quad \times t_{n^{\prime} l^{\prime} J^{\prime} M^{\prime}, n l J M}^{a_{j} a_{i}}\left(\boldsymbol{R}_{n}-\boldsymbol{R}_{m}+\boldsymbol{d}_{i}-\boldsymbol{d}_{j}\right),
\end{aligned}
$$

with

$$
\begin{aligned}
& t_{n^{\prime} l^{\prime} J^{\prime} M^{\prime}, n l J M}^{a_{j} a_{i}}\left(\boldsymbol{R}_{n}-\boldsymbol{R}_{m}+\boldsymbol{d}_{i}-\boldsymbol{d}_{j}\right) \\
& =\int \phi_{n^{\prime} \ell^{\prime} J^{\prime} M^{\prime}}^{a_{j}}(\boldsymbol{r})^{\dagger} \frac{V_{a_{j}}(\boldsymbol{r})+V_{a_{i}}\left(\boldsymbol{r}-\boldsymbol{R}_{n}+\boldsymbol{R}_{m}-\boldsymbol{d}_{i}+\boldsymbol{d}_{j}\right)}{2} \\
& \quad \times \phi_{n \ell J M}^{a_{i}}\left(\boldsymbol{r}-\boldsymbol{R}_{n}+\boldsymbol{R}_{m}-\boldsymbol{d}_{i}+\boldsymbol{d}_{j}\right) d^{3} r
\end{aligned}
$$

where $t_{n^{\prime} l^{\prime} J^{\prime} M^{\prime}, n l J M}^{a_{j} a_{i}}\left(\boldsymbol{R}_{n}-\boldsymbol{R}_{m}+\boldsymbol{d}_{i}-\boldsymbol{d}_{j}\right)$ denotes the relativistic hopping integral, and where $\phi_{n \ell J M}^{a_{i}}(\boldsymbol{r}), \bar{\varepsilon}_{n \ell J}^{a_{i}}(\boldsymbol{B}=0)$ and $\Delta \bar{\varepsilon}_{n l J M}^{a_{i}, \boldsymbol{d}_{i}}(\boldsymbol{B}=0)$ represent the relativistic atomic orbital, energy spectrum, and energy of the crystal field for the case of zero magnetic field, respectively. The quantities $n, \ell, J$, and $M$ are the principal, orbital, total angular momentum, and magnetic quantum numbers, respectively. From Eq. (5) it is found that matrix elements explicitly depend on the magnetic field. The third term in the diagonal elements is proportional to the magnetic field, which corresponds to the Zeeman energy term. The phase factor in the off-diagonal elements also depends on the magnetic field. Note that the relativistic hopping integral that is given by Eq. (6) is independent of the magnetic field. It is also found from Eq. (5) that relativistic effects are included in both $\bar{\varepsilon}_{n l J}^{a_{i}}(\boldsymbol{B}=0)+\Delta \bar{\varepsilon}_{n l J M}^{a_{i}, \boldsymbol{d}_{i}}(\boldsymbol{B}=0)$ and $t_{n^{\prime} l^{\prime} J^{\prime} M^{\prime}, n l J M}^{a_{j} a_{i}}\left(\boldsymbol{R}_{n}-\boldsymbol{R}_{m}+\boldsymbol{d}_{i}-\boldsymbol{d}_{j}\right)$. By diagonalizing the matrix elements of Eq. (5), the energy spectrum of the crystalline materials immersed in a uniform magnetic field are obtained.

In order to perform the actual calculations on the basis of the MFRTB method, we need the numerical value of $t_{n^{\prime} l^{\prime} J^{\prime} M^{\prime}, n l J M}^{a_{j} a_{i}}\left(\boldsymbol{R}_{n}-\boldsymbol{R}_{m}+\boldsymbol{d}_{i}-\boldsymbol{d}_{j}\right)$. In the case of the conventional TB approximation method (nonrelativistic and zero magnetic field case), the hopping integral is calculated by using TB parameters such as $s s \sigma, s p \sigma, p p \sigma$, and $p p \pi$ [23]. The results are summarized in tabular form, which is the socalled Slater-Koster table [23]. Similarly, also in the relativistic TB approximation method, $t_{n^{\prime} l^{\prime} J^{\prime} M^{\prime}, n l J M}^{a_{j} a_{i}}\left(\boldsymbol{R}_{n}-\boldsymbol{R}_{m}+\boldsymbol{d}_{i}-\right.$ $\boldsymbol{d}_{j}$ ) can be expressed in terms of relativistic TB parameters $K_{d}^{a_{j} a_{i}}\left(n^{\prime} \ell^{\prime} J^{\prime}, n \ell J\right)_{|M|}[11]$ that are defined by

$$
\begin{aligned}
K_{d}^{a_{j} a_{i}}\left(n^{\prime} \ell^{\prime} J^{\prime}, n \ell J\right)_{|M|} & \\
= & \int \phi_{n^{\prime} \ell^{\prime} J^{\prime} M^{\prime}}^{a j}(\boldsymbol{r})^{\dagger} \frac{V_{a_{j}}(\mathbf{r})+V_{a_{i}}\left(\boldsymbol{r}-R \boldsymbol{e}_{z}\right)}{2} \\
& \times \phi_{n \ell J M}^{a i}\left(\boldsymbol{r}-\boldsymbol{R e}_{z}\right) d^{3} r
\end{aligned}
$$

where $R=\left|\boldsymbol{R}_{n}-\boldsymbol{R}_{m}+\boldsymbol{d}_{i}-\boldsymbol{d}_{j}\right|$, and where $\boldsymbol{e}_{z}$ denotes the unit vectors along the $z$ axis. The subscript $d$ of $K_{d}^{a_{j} a_{i}}\left(n^{\prime} \ell^{\prime} J^{\prime}, n \ell J\right)_{|M|}$ stands for the dependence of the relativistic TB parameter on the distance $R$, i.e., if $R$ is equal to the distance between the nearest neighbor atoms, second-nearest neighbor atoms, and so on, then $d$ takes the value of 1 , $2, \ldots$, respectively [11]. The values of the relativistic TB parameters can be determined by utilizing the results of relativistic energy-band calculations of the zero magnetic field as the reference data, similar to the case of the nonrelativistic TB approximation method [24]. The relativistic version of the Slater-Koster table is given in Table I of Ref. [11]. For example, some of $t_{n^{\prime} l^{\prime} J^{\prime} M^{\prime}, n l J M}^{a_{j} a_{i}}\left(\boldsymbol{R}_{n}-\boldsymbol{R}_{m}+\boldsymbol{d}_{i}-\boldsymbol{d}_{j}\right)$ are given 
as follows [11]:

$$
\begin{aligned}
& t_{n^{\prime} 0 \frac{1}{2} \frac{1}{2}, n 0 \frac{1}{2} \frac{1}{2}}^{a_{j} a_{i}}\left(\boldsymbol{R}_{n}-\boldsymbol{R}_{m}+\boldsymbol{d}_{i}-\boldsymbol{d}_{j}\right)=K_{d}^{a_{j} a_{i}}\left(n^{\prime} 0 \frac{1}{2}, n 0 \frac{1}{2}\right)_{\frac{1}{2}}, \\
& t_{n^{\prime} 0 \frac{1}{2} \frac{1}{2}, n 0 \frac{1}{2}-\frac{1}{2}}^{a_{j} a_{n}}\left(\boldsymbol{R}_{n}-\boldsymbol{R}_{m}+\boldsymbol{d}_{i}-\boldsymbol{d}_{j}\right)=0 \\
& t_{n^{\prime} 0 \frac{1}{2} \frac{1}{2}, n 1 \frac{1}{2} \frac{1}{2}}^{a_{j} a_{i}}\left(\boldsymbol{R}_{n}-\boldsymbol{R}_{m}+\boldsymbol{d}_{i}-\boldsymbol{d}_{j}\right) \\
& \quad=z K_{d}^{a_{j} a_{i}}\left(n^{\prime} 0 \frac{1}{2}, n 1 \frac{1}{2}\right)_{\frac{1}{2}} \\
& t_{n^{\prime} 1 \frac{1}{2} \frac{1}{2}, n 1 \frac{3}{2} \frac{1}{2}}^{a_{j} a_{i}}\left(\boldsymbol{R}_{n}-\boldsymbol{R}_{m}+\boldsymbol{d}_{i}-\boldsymbol{d}_{j}\right) \\
& \quad=\frac{1}{2}\left(3 z^{2}-1\right) K_{d}^{a_{j} a_{i}}\left(n^{\prime} 1 \frac{1}{2}, n 1 \frac{3}{2}\right)_{\frac{1}{2}}
\end{aligned}
$$

where $x, y$, and $z$ represent the direction cosines of the vector $\boldsymbol{R}_{n}-\boldsymbol{R}_{m}+\boldsymbol{d}_{i}-\boldsymbol{d}_{j}$.

\section{B. Application of the MFRTB method to the simple cubic lattice immersed in the magnetic field}

We first explain the reason why we choose as the test system a hypothetical simple cubic lattice instead of realistic lattice structures. Since the purpose of this paper is to revisit the dHvA effect via the MFRTB method and to check the validity of the LK formula, we have to adopt as the target material of the actual calculations a simple system, for which the extremal cross section of the Fermi surface can be calculated exactly. Otherwise the resultant period that is calculated by the LK formula inevitably contains errors that come from the error of the extremal cross section of the Fermi surface, and therefore we cannot discuss the validity and accuracy of the LK formula itself. In this paper, we shall consider a hypothetical simple cubic lattice immersed in the magnetic field as a model system of the metal. In such a model, we suppose that each lattice point has one atom with one $s$ electron. Since the extremal cross section of the Fermi surface for more realistic lattice structure, which is usually calculated via the energy-band calculations, contains some kinds of errors inevitably [25], the application of the MFRTB method to the above-mentioned simple system is indispensable for discussing the validity and accuracy of the LK formula itself. After checking it, applying the MFRTB method to more realistic lattice structures is made possible. by

The magnitude of the magnetic field is assumed to be given

$$
B=2 \pi \frac{\hbar}{e a^{2}} \frac{p}{q},
$$

where $p$ and $q$ are relatively prime integers, and $a$ denotes the lattice constant. By utilizing the magnetic Bloch theorem that comes from the translational symmetry in a uniform magnetic field [11], we have the relation between the expansion coefficients:

$$
C_{\boldsymbol{k}}^{\xi}\left(\boldsymbol{t}_{n}+\text { Ia }_{y}\right)=e^{-i \boldsymbol{k} \cdot \boldsymbol{t}_{n}} C_{\boldsymbol{k}}^{\xi}\left(\text { Ia }_{y}\right),
$$

with $I=0,1,2, \ldots, q-1$. The translation vector $\boldsymbol{t}_{n}$ and wave vector $\boldsymbol{k}$ in Eq. (13) are defined by

$$
\boldsymbol{t}_{n}=n_{1} a \boldsymbol{e}_{x}+q n_{2} a \boldsymbol{e}_{y}+n_{3} a \boldsymbol{e}_{z},
$$

and

$$
\boldsymbol{k}=\frac{2 \pi}{a}\left(k_{1} \boldsymbol{e}_{x}+\frac{k_{2} \boldsymbol{e}_{y}}{q}+k_{3} \boldsymbol{e}_{z}\right)
$$

respectively, where $n_{1}, n_{2}$, and $n_{3}$ are integers, and where $k_{1}$, $k_{2}$, and $k_{3}$ are real numbers ranging from -0.5 to 0.5 . From Eq. (14), the "magnetic primitive cell" is defined by three primitive vectors:

$$
a \boldsymbol{e}_{x}, q a \boldsymbol{e}_{y}, \text { and } a \boldsymbol{e}_{z} .
$$

The corresponding "magnetic reciprocal lattice" is constructed from the following vectors:

$$
\frac{2 \pi}{a} \boldsymbol{e}_{x}, \quad \frac{2 \pi}{a q} \boldsymbol{e}_{y}, \quad \text { and } \quad \frac{2 \pi}{a} \boldsymbol{e}_{z} .
$$

The "magnetic first Brillouin zone" for the simple cubic lattice immersed in the magnetic field is presented in Fig. 1.

We consider only the hopping integrals between nearest neighbor atoms as done in the usual TB method [24]. Taking into consideration relativistic atomic orbitals with $(n, l, J, M)=(n, 0,1 / 2, \pm 1 / 2)$, and using Eq. (13), we can calculate matrix elements of the Hamiltonian, i.e., Eq. (5), and finally get the simultaneous equations for the expansion coefficients as

$$
\begin{aligned}
& {\left[\bar{\varepsilon}_{n 0 \frac{1}{2}}(\boldsymbol{B}=0)+\Delta \bar{\varepsilon}_{n 0 \frac{1}{2} M}(\boldsymbol{B}=0)+\frac{e B}{m} \hbar M\right.} \\
& \left.\quad+2 K_{1}\left(n 0 \frac{1}{2}, n 0 \frac{1}{2}\right)_{\frac{1}{2}}\left\{\cos \left(2 \pi k_{3}\right)+\cos \left(2 \pi\left(k_{1}+I \frac{p}{q}\right)\right)\right\}\right] \\
& \quad \times C_{\boldsymbol{k}}^{n 0 \frac{1}{2} M}\left(\text { Iae }_{y}\right)+K_{1}\left(n 0 \frac{1}{2}, n 0 \frac{1}{2}\right)_{\frac{1}{2}} \\
& \quad \times\left[C_{\boldsymbol{k}}^{n 0 \frac{1}{2} M}\left((I+1) a \boldsymbol{e}_{y}\right)+C_{\boldsymbol{k}}^{n 0 \frac{1}{2} M}\left((I-1) a \boldsymbol{e}_{y}\right)\right] \\
& =E(\boldsymbol{k}) C_{\boldsymbol{k}}^{n 0 \frac{1}{2} M}\left(\text { Ia }_{y}\right) .
\end{aligned}
$$

Note that the dependencies of $\bar{\varepsilon}_{n 0 \frac{1}{2}}(\boldsymbol{B}=0), \Delta \bar{\varepsilon}_{n 0 \frac{1}{2} M}(\boldsymbol{B}=0)$, $K_{1}\left(n 0 \frac{1}{2}, n 0 \frac{1}{2}\right)_{\frac{1}{2}}$, and $C_{\boldsymbol{k}}^{n 0 \frac{1}{2} M}$ on $a_{i}$ can be omitted, because the monoatomic crystal is considered. By solving the simultaneous equations, we have $2 q$ eigenvalues for each $\boldsymbol{k}$, and obtain

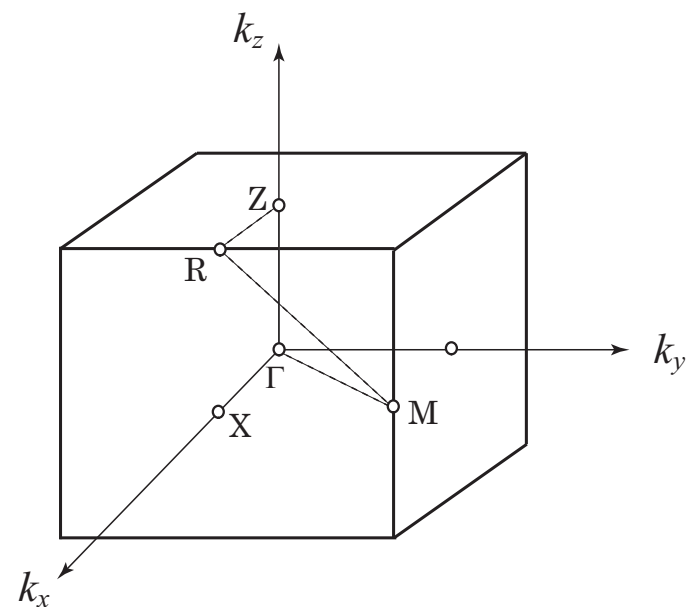

FIG. 1. Magnetic first Brillion zone for the simple cubic lattice immersed in a uniform magnetic field, together with some symmetry points, i.e., $\Gamma=\frac{2 \pi}{a}(0,0,0), Z=\frac{2 \pi}{a}(0,0,0.5), R=\frac{2 \pi}{a}(0.5,0,0.5)$, $X=\frac{2 \pi}{a}(0.5,0,0)$, and $M=\frac{2 \pi}{a}(0.5,0.5 / q, 0)$. 

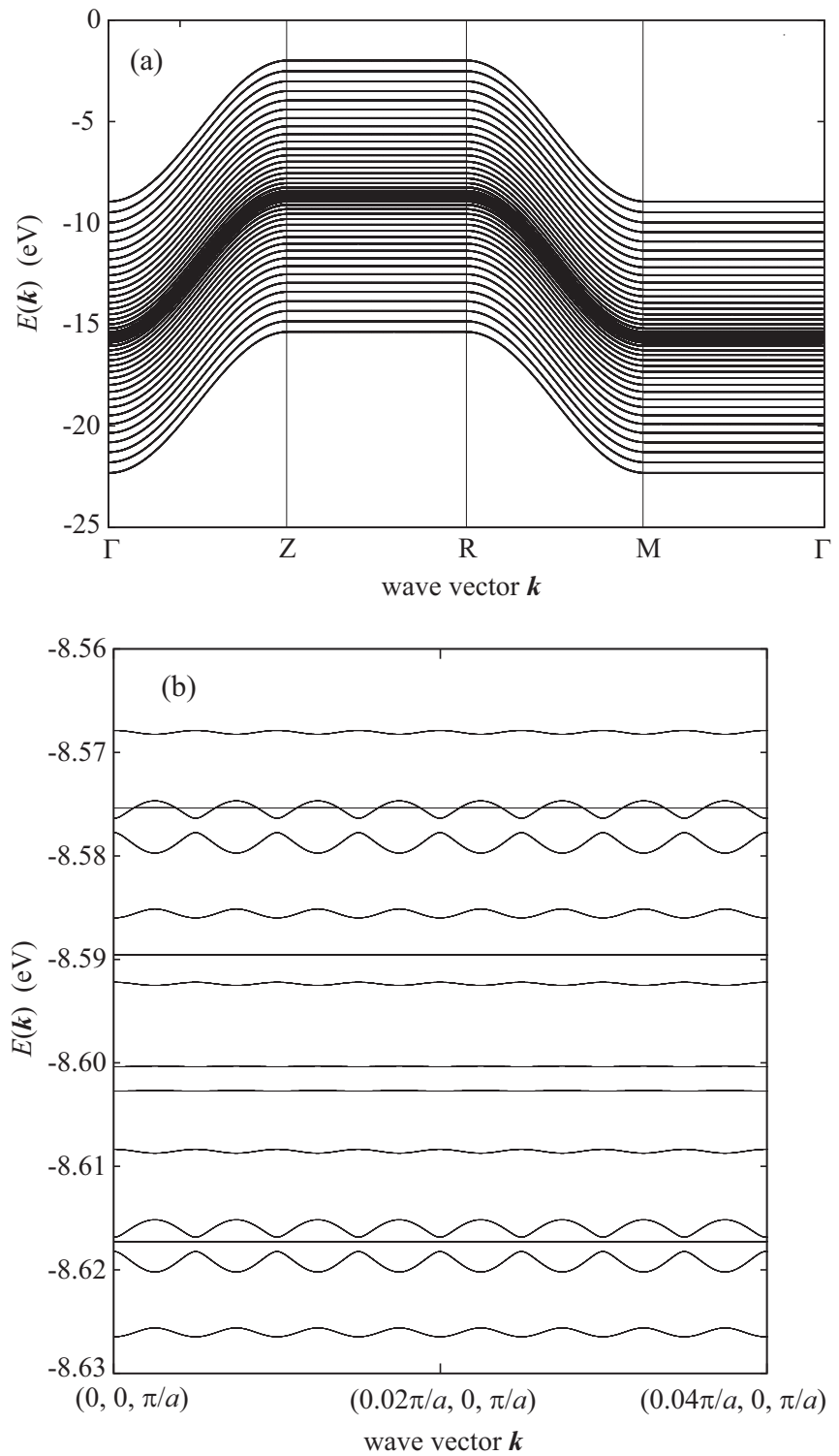

FIG. 2. (a) $E$ - $\boldsymbol{k}$ curves for the simple cubic lattice immersed in the magnetic field of $(p, q)=(10,401)$. (b) The magnified view of $\boldsymbol{E}$ - $\boldsymbol{k}$ curves along the Z-R line.

$E-\boldsymbol{k}$ curves in the presence of the magnetic field. Since the magnitude of relativistic TB parameters never affect discussions on the validity of the LK formula (Sec. IIIC), we here use the same values as we have used in Ref. [11]:

$$
\begin{aligned}
& \bar{\varepsilon}_{n 0 \frac{1}{2}}(\boldsymbol{B}=0)+\Delta \bar{\varepsilon}_{n 0 \frac{1}{2} M}(\boldsymbol{B}=0)=-12.1538(\mathrm{eV}), \\
& K_{1}\left(n 0 \frac{1}{2}, n 0 \frac{1}{2}\right)_{\frac{1}{2}}=-1.7391(\mathrm{eV}) .
\end{aligned}
$$

The results of actual calculations are presented in the next section.

\section{RESULTS AND DISCUSSIONS}

\section{A. Electronic structures for the simple cubic lattice immersed in a magnetic field}

Figure 2(a) shows $E$ - $\boldsymbol{k}$ curves of the simple cubic lattice immersed in a uniform magnetic field. Values of $p$ and $q$ are 10 and 401, respectively. Symbols in the horizontal axis correspond to the symmetry points in the magnetic first Brillouin zone that is drawn in Fig. 1. It is found from Fig. 2(a) that the energy little depends on the components of the wave vector perpendicular to the magnetic field (i.e., $k_{x}$ and $k_{y}$ ) and varies with the component parallel to the magnetic field (i.e., $k_{z}$ ). This means that the motion of electrons in the plane perpendicular to the magnetic field is essentially changed due to the Lorentz force. On the other hand, since the electron is not subjected to the Lorentz force in the $z$ direction, $E-\boldsymbol{k}$ curves remain with the relatively large bandwidth. Seeing the $E-\boldsymbol{k}$ curves macroscopically, they are positioned in the form of parallel lines with some energy spacing to each other, which seemingly look like the Landau levels. However, as shown in Fig. 2(b), each energy band has a small but definite width, which is hereafter called the fine structure of the $\boldsymbol{E}-\boldsymbol{k}$ curves [26]. Macroscopical shapes of the $E-\boldsymbol{k}$ curves would come from the fact that the orbital quantization contained in the LK formula inevitably emerges also in the calculation results of the MFRTB method, while the fine structure of the $E-\boldsymbol{k}$ curves is due to the periodic potential of the crystal [11]. Effects of this fine structure on the magnetic oscillation will be discussed in Sec. III D.

Figure 3(a) shows the dependence of the energy spectrum on the magnitude of the magnetic field in the case where the wave vector is restricted in the plane perpendicular to the magnetic field. In this calculation, $p$ changes from 1 to 401 with fixing $q$ at 401 . The characteristic gap structures, which are similar to Hofstadter's butterfly diagram [27], can be seen in Fig. 3(a). On the other hand, the characteristic gap structures are not found when the wave vector varies along the axis parallel to the magnetic field [Fig. 3(b)]. This is due to the strong dependence of the electron energy on $k_{z}$, which is shown in Fig. 2(a). In addition, the energy diagram shown in Figs. 3(a) and 3(b) split into two parts, which is due to the Zeeman term of Eq. (18). As can be seen in Fig. 3(a), the magnitude of such splittings become large as the magnetic field increases. Thus, the MFRTB method is regarded as the generalized method that includes Hofstadter's method [27].

\section{B. Density of states}

In order to show the oscillation of the total energy of the system immersed in a uniform magnetic field, we first calculate the DOS of the system. For this purpose, we use the following theorem that has been shown in the previous paper [11]:

(Theorem) "The total number of $\boldsymbol{k}$ points contained in the magnetic first Brillouin zone coincides with that of the magnetic primitive unit cells contained in the system."

We suppose that the number of magnetic primitive unit cells contained in the system is denoted by $N_{t_{n}}$. Since the volume of the magnetic primitive unit cell is given by $q a^{3}$ from Eq. (16), the volume of the system is equal to $q a^{3} N_{t_{n}}$. According to the theorem, there are $N_{t_{n}}$ points of $\boldsymbol{k}$ point in 

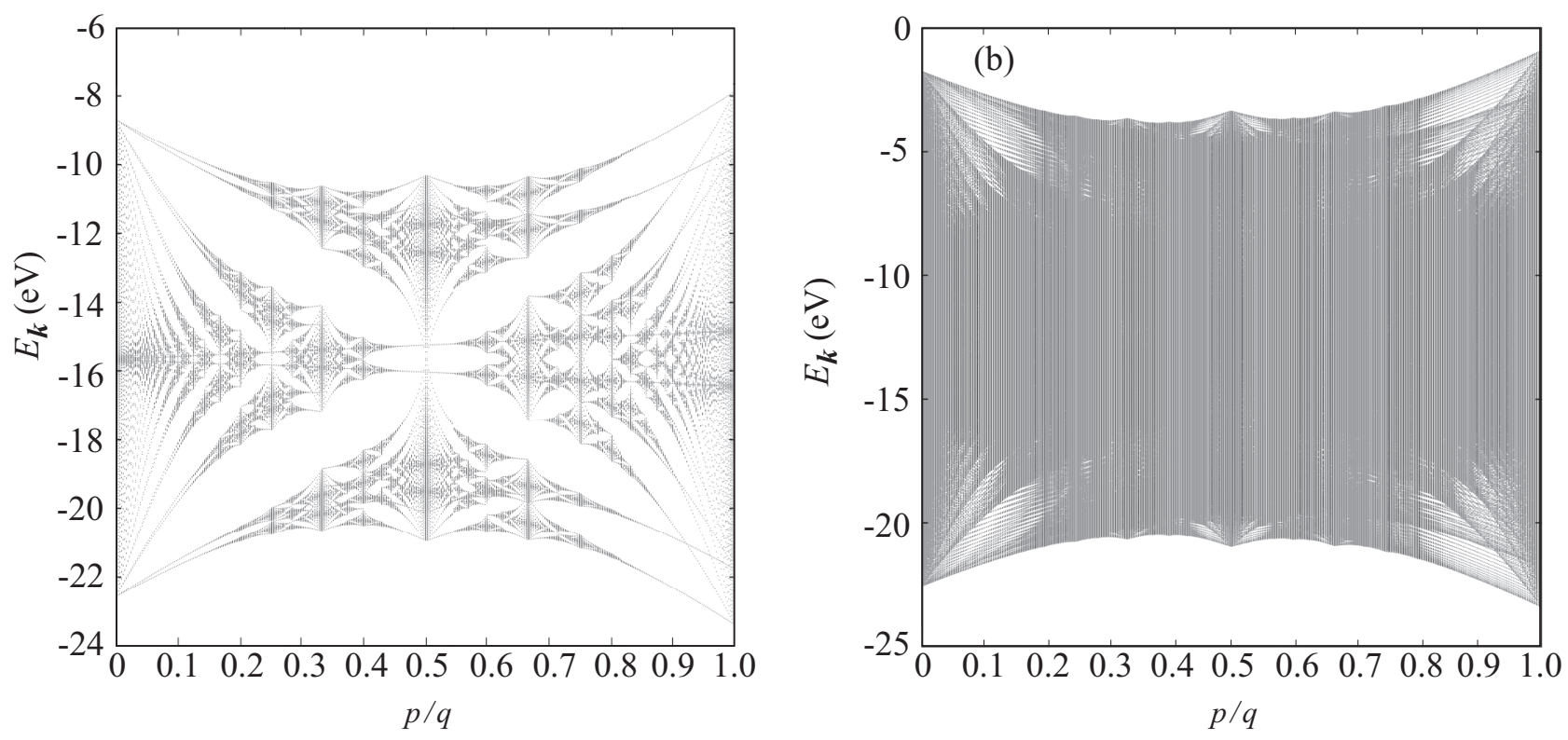

FIG. 3. Energy spectrum as a function of the magnitude of the magnetic field (a) in the cases of varying wave vectors in the $k_{x}-k_{y}$ plane of the magnetic first Brillouin zone and (b) in the case of varying wave vectors along the $k_{z}$ axis of the magnetic first Brillouin zone.

the magnetic first Brillouin zone. We assume that $\boldsymbol{k}$ points are distributed uniformly in the magnetic first Brillouin zone. This assumption would be reasonable because $\boldsymbol{k}$ points are distributed uniformly in the limit of the zero magnetic field. Under this assumption, we calculate $E(\boldsymbol{k})$ for each $\boldsymbol{k}$ point and calculate the total number of energy levels less than the energy $\varepsilon$, i.e, $N(\varepsilon)$. We obtain the DOS $D(\varepsilon)$ by differentiating $N(\varepsilon)$ with respect to $\varepsilon$, and by dividing it by the volume of the system $q a^{3} N_{t_{n}}$.

The DOSs for the simple cubic lattice immersed in a uniform magnetic field are shown in Figs. 4(a) and 4(b) for the cases of $(p, q)=(10,401)$ and $(p, q)=(1,1399)$, respectively. Figure 4(c) is the magnified view of Fig. 4(b). The value of $N_{t_{n}}$, which is used in actual calculations, is determined by requiring that the dependence of the total energy per unit volume on the size of the system is negligibly small. In these calculations, we take $8 q^{2} \times 10^{6}$ as $N_{t_{n}}$ [28]. It is found from Figs. 4(a)-4(c) that the DOS is analogous to that of the free electron immersed in a uniform magnetic field [29]. Namely, the DOS consists of two characteristic parts. One comes from $E-\boldsymbol{k}$ curves along the $k_{z}$ axis, the shape of which looks like the trapezoid that corresponds to the DOS of the simple cubic lattice for the zero magnetic field. The other comes from discretized energy levels, the shape of which looks like the delta function. It is also found in Fig. 4(c) that there are two types of energy splits. The large energy splitting corresponds to the orbital quantization, and the small one is due to the spin Zeeman splitting.

\section{Revisit of the dHvA effect via the MFRTB method}

In order to calculate the total energy, the Fermi energy must be estimated. In the present model system, one lattice point has one atom with one $s$ electon, so that there exists one electron in each lattice point. Since it has $q$ lattice points, the magnetic primitive unit cell contains $q$ electrons. Since $N_{t_{n}}$ magnetic primitive unit cells are contained in the system, the total number of electrons in the model system is given by $q N_{t_{n}}$. According to the theorem mentioned in the previous subsection, the total number of $\boldsymbol{k}$ points is equal to $N_{\boldsymbol{t}_{n}}$. Since $2 q$ energy levels are calculated for each $\boldsymbol{k}$ point (Sec. III), a total of $2 q N_{t_{n}}$ energy levels is obtained in the magnetic first Brillouin zone. Therefore, the lower half of energy levels are occupied by electrons, which corresponds to the valence bands. Using this fact, the Fermi energy can be estimated. The Fermi energy estimated is about $-12.538(\mathrm{eV})$ for each case.

Figures 5 and 6 show the dependence of the total energy $\left(E_{\text {total }}\right)$ on the inverse of the magnetic field ranging from 9.78 to 9.90 (T) (Fig. 5) and from 43.5 to 45.7 (T) (Fig. 6), respectively. Oscillatory behavior of the total energy with respect to the magnetic field is clearly observed. The magnetization can be calculated by taking the differential of the total energy with respect to the magnetic field, where we use the cubic-spline interpolation technique. The magnetic-field dependencies of the magnetization $[M(B)]$ are shown in Figs. 7 and 8. In Fig. 8, some spikelike peaks of the magnetization can be found in addition to the global oscillation. Hereafter, we discuss the global oscillation of the magnetization. Concerning the spikelike peaks, we will discuss in the subsequent subsection.

The period of the global oscillation of the magnetization can be calculated by the Fourier transformation of the waveform of the magnetization. The evaluated values of periods corresponding to Figs. 7 and 8 are $3.87 \times 10^{-4}(1 / \mathrm{T})$ and $3.85 \times 10^{-4}(1 / \mathrm{T})$, respectively (Table I).

On the other hand, according to the LK formula, the period of the oscillation of the magnetization is related to the extremal cross-section area of the Fermi surface $A_{\text {ext }}$ for the zero magnetic field system, which is given by

$$
\Delta\left(\frac{1}{B}\right)=\frac{2 \pi e}{\hbar A_{\mathrm{ext}}} .
$$



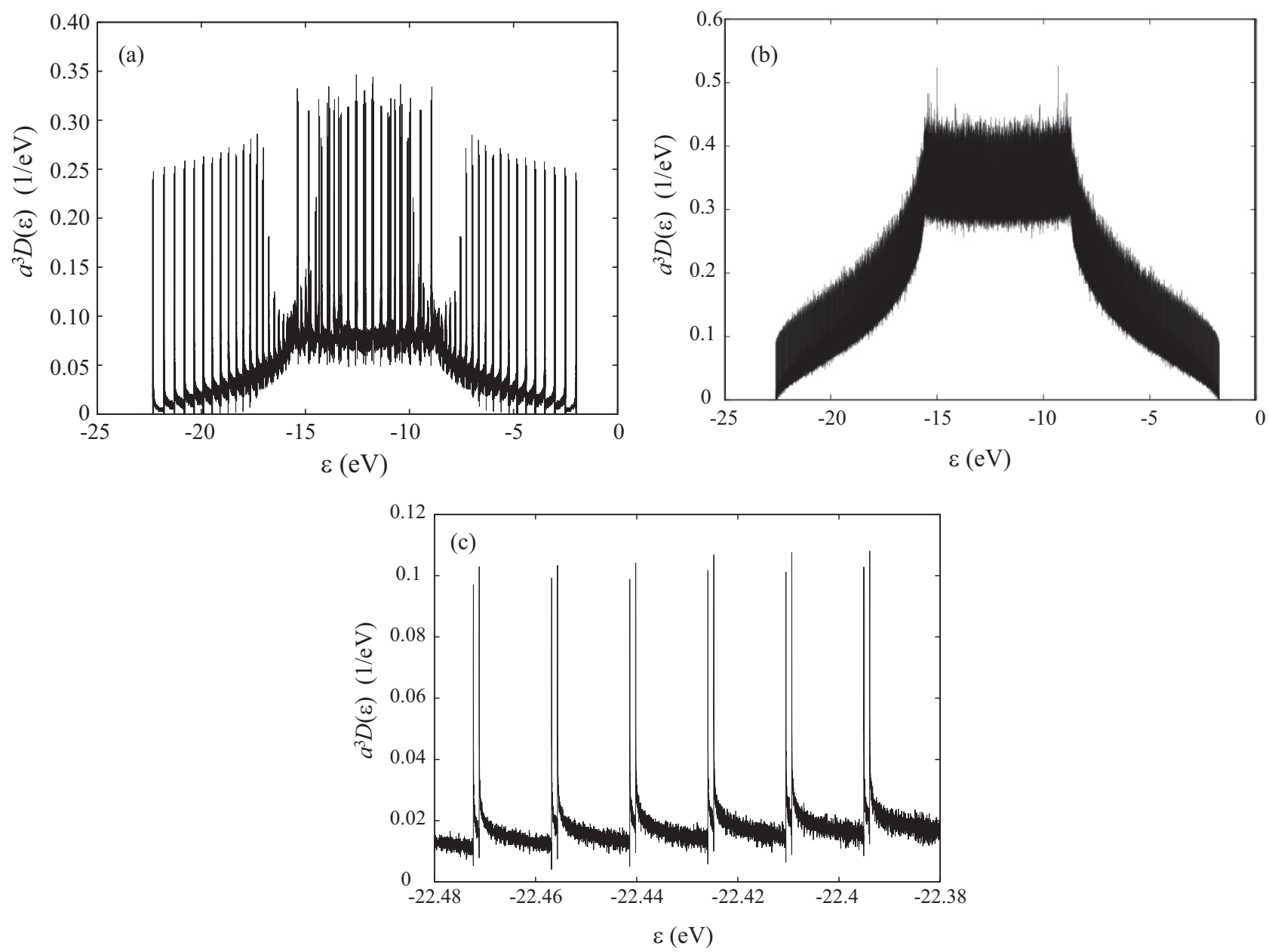

FIG. 4. Density of states in the cases of $(a)(p, q)=(10,401)$ and (b) $(p, q)=(1,1399)$. The magnified view of (b) is shown in (c).

In the case of the simple cubic lattice, the extremal cross sections of the Fermi surface exist at the boundary of the Brillouin zone [30]. Two kinds of the Fermi surface exist; one is the electron sheet that is centered at the $\mathrm{X}$ point of the Brillouin zone [31], and the other is the hole sheet that is centered at the $\mathrm{M}$ point of the Brillouin zone [31]. They have the same cross-section areas, which results in the degenerate magnetic oscillations of the dHvA effect. The sizes of the Fermi surface sheets are dependent on the TB parameters of the zero magnetic field. The use of the same values as Eq. (19) allows comparison of the results of the LK formula with those of the MFRTB method. Using Eq. (19), the Fermi surface of the simple cubic lattice is easily obtained. Then using Eq. (20), the period by the LK formula is estimated as $3.858 \times 10^{-4}(1 / \mathrm{T})$, which is quite close to the periods that are obtained from the
MFRTB method (Table I). This means that the LK formula, which is based on the Bohr-Sommerfeld quantization rule and semiclassical equation, is a good approximation in the magnetic field less than about $46(\mathrm{~T})$.

\section{Additional oscillation peaks of the magnetization}

As mentioned in the previous subsection, some spikelike peaks of the magnetization can be found in Fig. 8 in addition to the global oscillation that is consistent with the conventional LK formula. Taking into consideration the fact that the LK formula relates the origin of the magnetic oscillation with the extremal cross section of the Fermi surface, additional oscillation peaks of the magnetization cannot be explained by the LK formula. These additional peaks are thought to be due to

TABLE I. Periods of the oscillation of the magnetization.

\begin{tabular}{lccc}
\hline \hline & & Period $(1 / \mathrm{T})$ & \\
\hline & $B=9.78-9.90(\mathrm{~T})$ & $B=43.5-45.7(\mathrm{~T})$ & $B=538.69-3328(\mathrm{~T})$ \\
LK formula & $3.858 \times 10^{-4}$ & Same as on the left & Same as on the left \\
MFRTB method & $3.87 \times 10^{-4}$ & $3.85 \times 10^{-4}$ & $3.98 \times 10^{-4}$ \\
\hline \hline
\end{tabular}




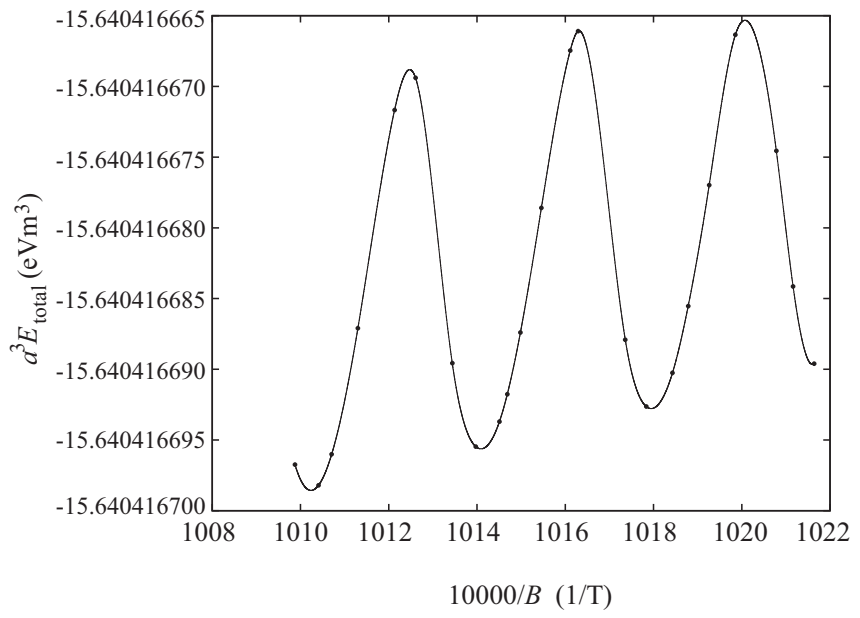

FIG. 5. Dependence of the total energy on the inverse of the magnitude of the magnetic field ranging from 9.78 (T) to $9.80(\mathrm{~T})$.

the effect of the periodic potential of the crystal. This is because the Landau levels, which are obtained for the case of the zero periodic potential, do not cause such additional oscillation peaks of the magnetization $[4,6]$. The periodic potential of the crystal also results in the fine structure of $E-\boldsymbol{k}$ curves that is shown in Fig. 2(b). Therefore, the fine structure would produce additional oscillation peaks that cannot be related to the Fermi surface via the LK formula. Furthermore, the effect of the energy band widening in the $k_{x}-k_{y}$ plane, which corresponds to the fine structure of $\boldsymbol{E}-\boldsymbol{k}$ curves, becomes remarkable in the high magnetic field due to the increase of the electron hopping with the magnitude of the magnetic field [11]. This is the reason why the additional oscillation peaks of the magnetization are clearly seen in Fig. 8 while they are not clearly seen in Fig. 7. Of course, it is expected that more numbers of spikelike peaks may appear in the magnetization curves if the intervals of the magnetic field are narrowed in the calculations.

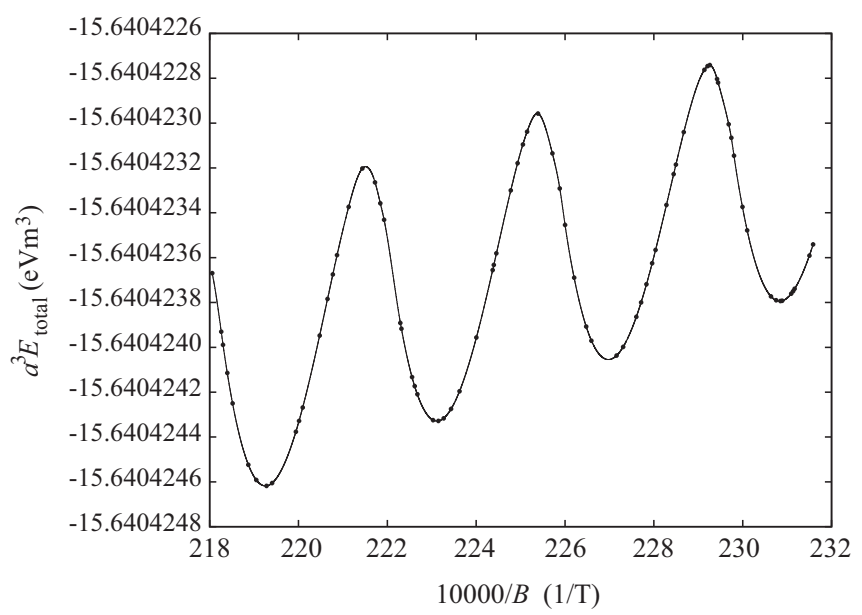

FIG. 6. Dependence of the total energy on the inverse of the magnitude of the magnetic field ranging from 43.5 (T) to 45.7 (T).

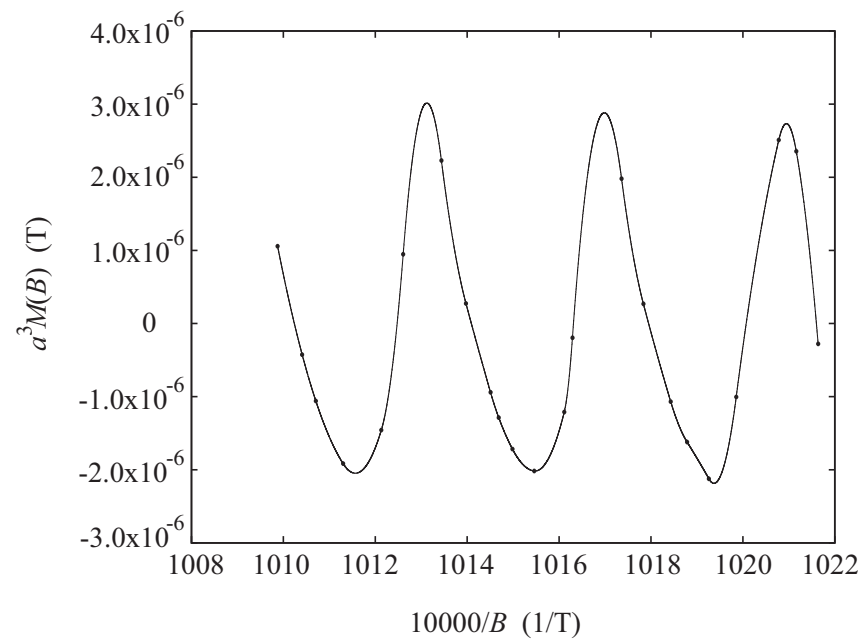

FIG. 7. Dependence of the magnetization on the inverse of the magnitude of the magnetic field ranging from 9.78 (T) to $9.80(\mathrm{~T})$.

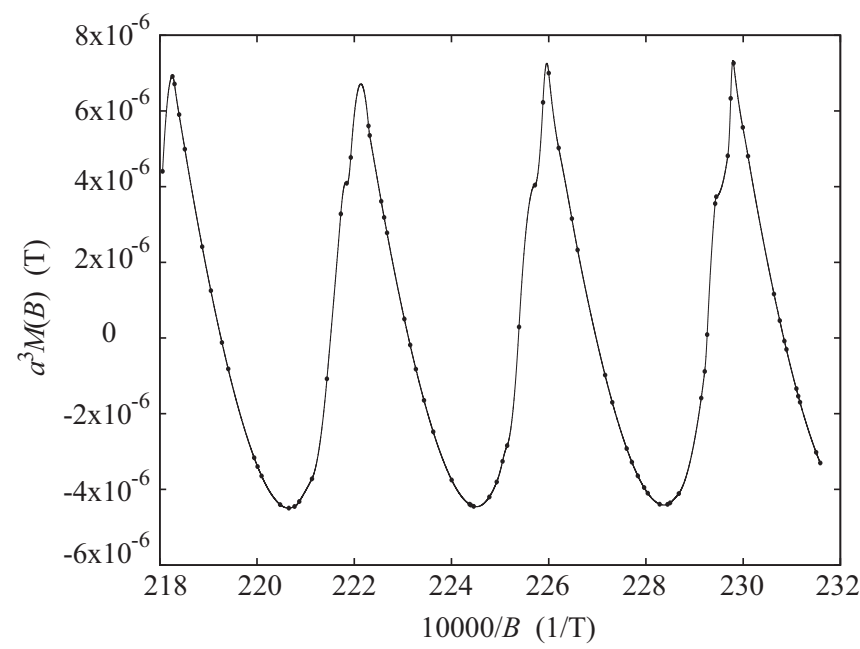

FIG. 8. Dependence of the magnetization on the inverse of the magnitude of the magnetic field ranging from 43.5 (T) to 45.7 (T).

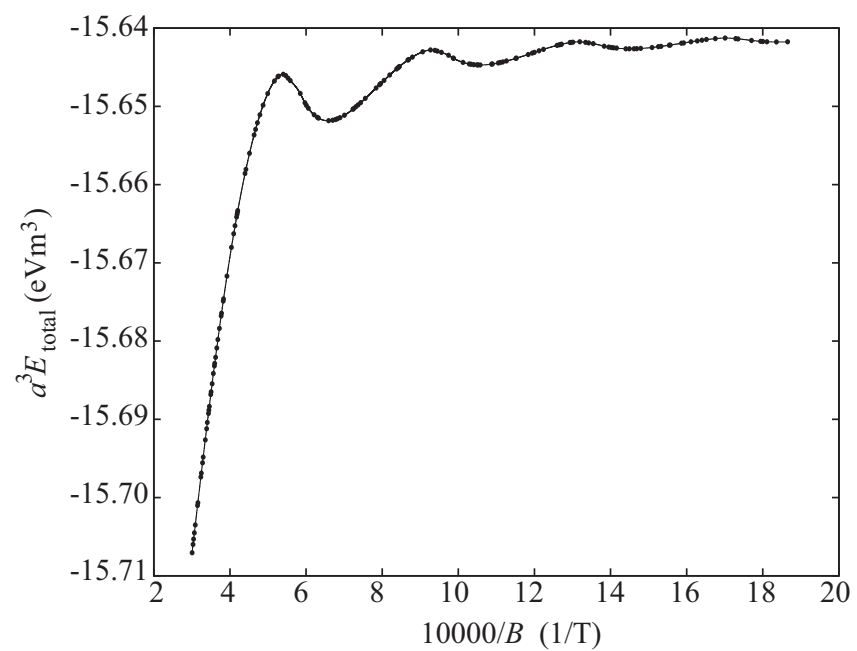

FIG. 9. Dependence of the total energy on the inverse of the magnitude of the magnetic field ranging from 538.69 (T) to 3328 (T). 


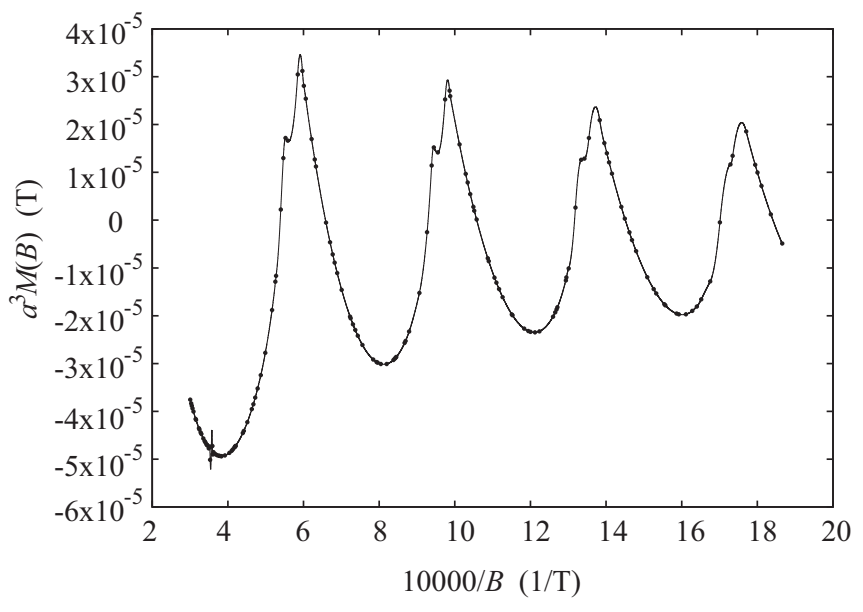

FIG. 10. Dependence of the magnetization on the inverse of the magnitude of the magnetic field ranging from 538.69 (T) to 3328 (T).

\section{E. Extremely high magnetic field}

For reference, we shall consider the case of the extremely high magnetic field. In the extremely high magnetic field ranging from 538.69(T) to 3328(T), the magnetic oscillations of the total energy and magnetization are calculated as shown in Figs. 9 and 10, respectively. Additional oscillation peaks, which are discussed in the previous subsection, are found in Fig. 10 more clearly than in Fig. 8. Another noticeable thing in the extremely high magnetic field is that the period of the global oscillation increases. Specifically, it is estimated as $3.98 \times 10^{-4}(1 / \mathrm{T})$, which deviates from the LK formula's by about $3 \%$ (Table I). This would possibly be caused by the limit of the validity of the LK formula. We shall discuss the reason bellow.

As mentioned in Sec. I, the LK formula is based on the Bohr-Sommerfeld quantization rule that is valid for the energy levels with a fairly high quantum number [10]. The maximum quantum number is roughly estimated by the ratio $\varepsilon_{F} / \hbar \omega_{c}$, where $\varepsilon_{F}$ and $\omega_{c}$ are the Fermi energy and cyclotron frequency, respectively [10]. This ratio becomes the order of $10^{3}$ in the case of $B \sim 10(\mathrm{~T})$, in which case the results of the MFRTB method certainly agree with those calculated from the LK formula, as mentioned in the previous subsection. On the other hand, in the case of $B \sim 10^{3}(\mathrm{~T})$, the ratio is about 10, which seems to be too small for the correctness of the Bohr-Sommerfeld quantization rule, as expected from the present results (Table I). The ratio $\varepsilon_{F} / \hbar \omega_{c}$ may become a practical and quantitative indication of whether the LK formula holds well or not. It should be noted that, since the Fermi energy generally increases with the electron density, there is a possibility that the incorrectness of the LK formula would become obvious even in less than $10^{3}(\mathrm{~T})$ for the metallic system with the low electron density.

\section{CONCLUDING REMARKS}

The MFRTB method is a useful first-principles calculation method that enables us to directly obtain electronic structures and magnetic properties of materials immersed in the magnetic field. In this paper, the MFRTB method is applied to the simple cubic lattice immersed in a uniform magnetic field. Oscillations of the total energy and magnetization (dHvA effect) are successfully revisited in a first-principles way by the MFRTB method. Although the conventional LK formula for the dHvA effect is a good approximation in the experimentally available magnetic field (around 9.8T and 46T), the MFRTB method is capable of becoming a useful method to describe the magnetic oscillations without the assumptions contained in the LK formula. Especially in the high magnetic field, the additional magnetic oscillation, which cannot be explained by the LK formula, is found by the MFRTB method. This additional magnetic oscillation may come from the fine structure of $E-\boldsymbol{k}$ curves, which is first revealed through the MFRTB method.

The present work may become an important milestone toward revisiting the $\mathrm{dHvA}$ oscillations of more realistic lattice structures by means of the MFRTB method. For example, when we apply the MFRTB method to more realistic lattice structures, magnetic oscillations will be obtained in a similar way to the present case (a hypothetical simple cubic lattice). Namely, by reference to the above-mentioned knowledge obtained from the present work, the LK formula is expected to give a good approximation for the period of the main oscillation in the low magnetic field region. Therefore, if there is a discrepancy between the period that is calculated by the MFRTB method and that of the LK formula, it can be concluded that this discrepancy comes from the error of the extremal cross section of the Fermi surface. Here note that the calculated value of the extremal cross section for realistic lattice structures inevitably contains some kinds of errors [25] because it is estimated by means of the energy-band calculations. In addition, if additional fine oscillations besides the main oscillation are observed in experiments, we can say that such fine oscillations do not always come from the errors of the extremal cross section of the calculated Fermi surface but may come from the fine structures of $\boldsymbol{E}-\boldsymbol{k}$ curves obtained from the MFRTB method. These kinds of discussions for realistic lattice structures are made possible only if we have checked the validity and accuracy of the LK formula by using the present simple model (hypothetical simple cubic lattice) in which the Fermi surface is exactly given.

The MFRTB method is applicable to actual crystalline materials immersed in the magnetic field. Although the target system of the present work is a three-dimensional lattice, the MFRTB method is, of course, applicable to twodimensional model systems, for which the dHvA oscillations have been investigated theoretically [32-39] by using the Peierls substitution or the Peierls phase [40]. The next issue is the first-principles investigations of the $\mathrm{dHvA}$ oscillations not only for the two-dimensional model but also for the actual three-dimensional metals by means of the MFRTB method.

\section{ACKNOWLEDGMENT}

This work was partially supported by Grant-in-Aid for Scientific Research (Grants No. 26400354 and No. 26400397) of Japan Society for the Promotion of Science. 
[1] W. J. de Haas and P. M. van Alphen, Proc. Netherlands Roy. Acad. Sci. 33, 680 (1930).

[2] W. J. de Haas and P. M. van Alphen, Proc. Netherlands Roy. Acad. Sci. 33, 1106 (1930).

[3] W. J. de Haas and P. M. van Alphen, Proc. Netherlands Roy. Acad. Sci. 35, 454 (1932).

[4] D. Shoenberg, Magnetic Oscillation in Metals (Cambridge University Press, Cambridge, 1984).

[5] L. D. Landau, Z. Phys. 64, 629 (1930).

[6] J. Kübler, in Theory of Itinerant Electron Magnetism (Oxford University Press, Oxford, 2000), Chap. 1.

[7] L. Onsager, Phil. Mag. 43, 1006 (1952).

[8] I. M. Lifshitz and A. M. Kosevich, Zh. Eksp. Teor. Fiz. 29, 730 (1955) [Sov. Phys. JETP 2, 636 (1956)].

[9] For example, in the case of $f$-electron materials, see M. R. Norman and D. D. Koelling, in Handbook on the Physics and Chemistry of Rare Earths, edited by K. A. Gschneidner Jr., L. Eyring, G. H. Lander, and G. R. Choppin (North-Holland, Amsterdam, 1993), Vol. 17, Chap. 110; Y. Onuki and A. Hasegawa, in Handbook on the Physics and Chemistry of Rare Earths, edited by K. A. Gschneidner Jr. and L. Eyring (North-Holland, Amsterdam, 1995), Vol. 20, Chap. 135.

[10] N. W. Ashcroft and N. D. Mermin, in Solid State Physics (W. B. Saunders, Philadelphia, 1976), Chap. 14.

[11] K. Higuchi, D. B. Hamal, and M. Higuchi, Phys. Rev. B 91, 075122 (2015).

[12] T. Goto, H. Yamada-Kaneta, Y. Saito, Y. Nemoto, K. Sato, K. Kakimoto, and S. Nakamura, J. Phys. Soc. Jpn. 75, 044602 (2006).

[13] S. Baba, T. Goto, Y. Nagai, M. Akatsu, H. Watanabe, K. Mitsumoto, T. Ogawa, Y. Nemoto, and H. Yamada-Kaneta, J. Phys. Soc. Jpn. 80, 094601 (2011).

[14] K. Okabe, M. Akatsu, S. Baba, K. Mitsumoto, Y. Nemoto, H. Yamada-Kaneta, T. Goto, H. Saito, K. Kashima, and Y. Saito, J. Phys. Soc. Jpn. 82, 124604 (2013).

[15] K. Mitsumoto, M. Akatsu, S. Baba, R. Takasu, Y. Nemoto, T. Goto, H. Yamada-Kaneta, Y. Furumura, H. Saito, K. Kashima, and Y. Saito, J. Phys. Soc. Jpn. 83, 034702 (2014).

[16] G. Vignale and M. Rasolt, Phys. Rev. Lett. 59, 2360 (1987).

[17] G. Vignale and M. Rasolt, Phys. Rev. B 37, 10685 (1988).

[18] K. Higuchi and M. Higuchi, Phys. Rev. B 74, 195122 (2006); 75, 159902(E) (2007).

[19] M. Higuchi and K. Higuchi, Phys. Rev. B 75, 195114 (2007).

[20] M. Higuchi and K. Higuchi, Phys. Rev. A 81, 042505 (2010).

[21] M. Higuchi and A. Hasegawa, J. Phys. Soc. Jpn. 66, 149 (1997).

[22] M. Higuchi and A. Hasegawa, J. Phys. Soc. Jpn. 67, 2037 (1998).

[23] J. C. Slater and G. F. Koster, Phys. Rev. 94, 1498 (1954).
[24] For example, see, W. A. Harrison, in Elementary Electronic Structure, Revised ed. (World Scientific, London, 2004), Chap. 1.

[25] They are caused by (i) treatment of the exchange and correlation effects, (ii) choice of the basis function in expanding the Bloch states, (iii) physical meanings of the single-particle spectra, and so on.

[26] A similar fine structure has been observed also in the crystalline silicon immersed in the magnetic field [11].

[27] D. R. Hofstadter, Phys. Rev. B 14, 2239 (1976).

[28] The details of how to determine the total number of $\boldsymbol{k}$ points are as follows. We divide the $k_{y}$ axis into 200 intervals within the magnetic first Brillouin zone. Since the magnetic first Brillouin zone shrinks by the factor $1 / q$ in the $k_{y}$ direction (Fig. 1), the resultant interval of $\boldsymbol{k}$ points $(\Delta k)$ is given by $\frac{2 \pi}{q a} \times \frac{1}{200}$. Since $\boldsymbol{k}$ points are assumed to be distributed uniformly in the magnetic first Brillouin zone (Sec. III B), the numbers of $\boldsymbol{k}$ points along the $k_{x}$ and $k_{z}$ axes are equal to $\frac{2 \pi}{a \Delta k}(=200 q)$. Consequently, the total number of $\boldsymbol{k}$ points is given by $200 q \times 200 q \times 200=$ $8 q^{2} \times 10^{6}\left(=N_{t_{n}}\right)$. If we change the division number (200), then the volume of the system that is given by $q a^{3} N_{t_{n}}$ also changes. The division number is determined by requiring that the dependence of the total energy per unit volume on the volume of the system is negligibly small.

[29] For example, see, J. Singleton, in Band Theory and Electronic Properties of Solids (Oxford University Press, New York, 2001), Chap. 8.

[30] C. Kittel, in Introduction to Solid State Physics, 8th ed. (John Wiley \& Sons, New York, 2005), Chap. 9.

[31] C. J. Bradley and A. P. Cracknell, in The Mathematical Theory of Symmetry in Solids (Clarendon Press, Oxford, 1972), Chap. 3.

[32] K. Machida, K. Kishigi, and Y. Hori, Phys. Rev. B 51, 8946 (1995).

[33] P. S. Sandhu, J. H. Kim, and J. S. Brooks, Phys. Rev. B 56, 11566 (1997).

[34] S. Y. Han, J. S. Brooks, and J. H. Kim, Phys. Rev. Lett. 85, 1500 (2000).

[35] O. Gat and J. E. Avron, Phys. Rev. Lett. 91, 186801 (2003).

[36] M. Taut, H. Eschrig, and M. Richter, Phys. Rev. B 72, 165304 (2005).

[37] V. M. Gvozdikov and M. Taut, Phys. Rev. B 75, 155436 (2007).

[38] W. H. Xu, L. P. Yang, M. P. Qin, and T. Xiang, Phys. Rev. B 78, 241102 (2008).

[39] K. Kishigi and Y. Hasegawa, Phys. Rev. B 90, 085427 (2014).

[40] M. P. Marder, in Condensed Matter Physics (John Wiley \& Sons, New York, 2000), Chap. 25. 